\title{
Pilot study of azithromycin in the treatment of genital donovanosis
}

\author{
Francis J Bowden, Jackie Mein, Catherine Plunkett, Ivan Bastian
}

\begin{abstract}
Objectives: To determine the effectiveness of azithromycin, an azalide antibiotic with long tissue half-life, in a pilot study of patients with genital donovanosis in the Northern Territory, Australia.

Design: Patients with histologically confirmed donovanosis were randomised to receive one of two open-label azithromycin dosage regimens: Regimen A-1.0 g once weekly for 4 weeks; or Regimen B-500 mg daily for 7 days. Patients were assessed at 6 weeks and classified as either "cured", "improved" or "failed".

Results: Seven patients received regimen A and 4 received regimen B. Six weeks after commencing treatment the genital ulcers of four patients receiving regimen $A$ and one patient receiving regimen $\mathbf{B}$ had healed; the lesions of the other six patients ( 3 in each regimen) were "improved". No patient failed to respond and no significant adverse reaction was recognised. The eleven patients were reviewed after completing the six-week trial; all lesions had re-epithelialised without further antibiotic treatment, no relapses had occurred, the longest follow-up period being seven months. A further 17 patients with donovanosis who were unable to meet the entry criteria were also treated successfully with azithromycin during the study period.

Conclusions: This is the first time that azithromycin has been shown to have clinical activity against donovanosis. Poor compliance with prolonged courses of antibiotics is one of the major barriers to control of the disease. Intermittent or short-course therapy, made possible by the long tissue half-life of the drug, could facilitate control of donovanosis in endemic populations if the high cost of medication can be addressed.

(Genitourin Med 1996;72:17-19)
\end{abstract}

Keywords: donovanosis; azithromycin; genital

\section{Introduction}

Donovanosis is an indolent genital ulcerative disease seen in Third and Fourth World populations, including Australian Aborigines. ${ }^{1}$ In Australia the disease is grossly under-notified: official notification data may under-represent the true prevalence of disease by a factor of 3 or more. ${ }^{2}$ The disease is presumably sexually transmitted and may lead to genital deformity or a life-threatening disseminated infection. More importantly, genital ulcerative diseases such as donovanosis may potentiate transmission of the human immunodeficiency virus (HIV). ${ }^{3-5}$

Histopathological examination of a lesion smear or biopsy remains the only laboratory investigation for donovanosis ${ }^{1}$ because the causative organism, Calymmatobacterium granulomatis (an intracellular gram-negative bacillus), cannot be cultured by routine microbiological techniques. Elsewhere, we have described the molecular characterisation of Cal granulomatis which represents the first step in developing better diagnostics for this genital ulcerative disease. ${ }^{6}$

Tetracyclines, erythromycin, co-trimoxazole, chloramphenicol ${ }^{1}$ and ceftriaxone ${ }^{7}$ successfully treat donovanosis if taken daily (or more frequently) for several weeks. However, primary treatment failures and relapse are common because of poor adherence to these prolonged daily regimens. A reliable and convenient treatment is therefore required to control donovanosis and hence help limit HIV transmission. ${ }^{5}$ We addressed this problem by conducting the first evaluation of azithromycin, a new long-acting azalide antibiotic with gram-negative activity, ${ }^{8}$ in the treatment of donovanosis.

\section{Methods}

Between June 1994 and March 1995, consenting patients between 16 and 70 years of age presenting or referred with donovanosis to the Communicable Disease clinics in Darwin or Katherine in the Northern Territory were enrolled in an open, non-comparative trial approved by the relevant Institutional Ethics Committee. Donovanosis was diagnosed clinically and confirmed by histological examination of lesion biopsies and/or smears. ${ }^{9}$ Baseline biochemical and haematological tests were also performed, as were investigations for other sexually transmitted diseases (STDs). Subjects were excluded if they had received another antibiotic in the preceding month or if pregnancy, lactation, allergy or systemic disease precluded azithromycin treatment.

Eligible patients were randomly assigned to receive a weekly $1 \mathrm{~g}$ dose of azithromycin for four weeks (regimen A) or $500 \mathrm{mg}$ once daily for seven days (regimen B). All treatments were supervised. Progress was assessed over the following six weeks by weekly examination of the number, size and appearance of the genital lesion(s) (and by photography if the patient consented). The clinical outcome was 
Patient data

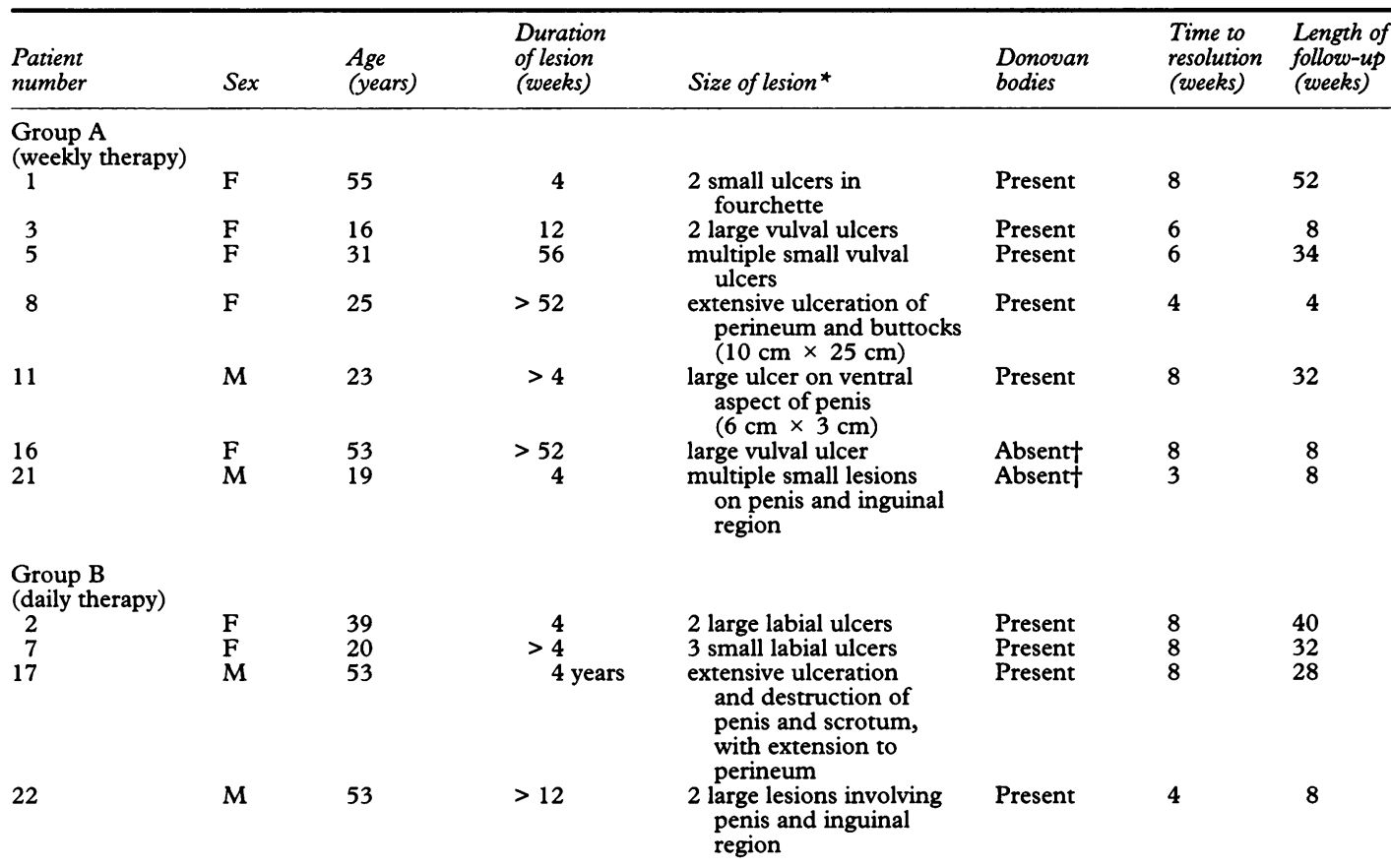

${ }^{\star}$ Large lesion defined as $>4 \mathrm{~cm}^{2}$; small lesion defined as $\leqslant 4 \mathrm{~cm}^{2}$.

†The following case definition for donovanosis was used: the presence of typical clinical appearances in conjunction with characteristic histological features. (In the absence of Donovan bodies a diagnosis could be made in the presence of the following: marked marginal epithelial hyperplasia, a dense plasma cell infiltrate in the dermis, neutrophilic abscesses, some large mononuclear cells but a paucity of lymphocytes ${ }^{9}$ ).

then defined as "cured" (that is, ulcer(s) completely healed), "improved" (that is, lesion(s) reduced in size/number and re-epithelialising) or "failed" (that is, ulcers unchanged or worse). Any adverse events were recorded and the biochemical and haematological tests repeated two and four weeks after treatment commenced.

\section{Results}

Results were evaluable for four men and seven women (mean age, 35.2 years; range, 16-55) with histologically-confirmed genital donovanosis. The clinical findings are summarised in the table. The duration of disease ranged between one month and four years. Histological demonstration of Donovan bodies (that is, the causative organism) in ulcer biopsies verified the clinical diagnosis in nine subjects; the other two evaluable patients had typical genital lesions and their biopsy specimens showed all the histological features of donovanosis ${ }^{9}$ except for Donovan bodies. Another five patients with genital ulcers clinically suggestive of donovanosis were enrolled but then discharged from the trial when their diagnosis could not be unequivocally confirmed by histology.

Of the 11 evaluable patients, seven received regimen $\mathrm{A}$ and four received regimen $\mathrm{B}$. Six weeks after commencing treatment the genital ulcers of four patients receiving regimen $A$ and one patient receiving regimen $B$ had healed; the lesions of the other six patients (three regimen-A and three regimen-B) were "improved" (follow-up beyond six weeks showed all such lesions re-epithelialised without further antibiotic treatment). No patient failed to respond and no significant adverse reaction was recognised (two patients complained of mild nausea while two others developed vaginal candidiasis). The eleven patients were reviewed after completing the six-week trial; no relapses had occurred, the longest follow-up period being 12 months. Furthermore, donovanosis remained the clinical diagnosis for three of the five patients discontinued from the trial because of inconclusive histological findings. All three were successfully treated with azithromycin.

\section{Discussion}

This pilot study provides clinical evidence that azithromycin effectively treats donovanosis. Cal granulomatis is not easily cultured (the last reported isolation was in 1962), ${ }^{10}$ therefore in vitro sensitivity testing cannot be used to support our findings. However, azithromycin does have greater in vitro activity against other gram-negative organisms than erythromycin, ${ }^{8}$ a proven treatment for donovanosis. Our cohort must be followed for 6-18 months to exclude relapse. ${ }^{1}$ Additional studies are required to confirm our results, to determine the optimal dose and duration of therapy, and to compare the cost-effectiveness of azithromycin with standard therapies.

$\mathrm{O}^{\prime}$ Farrell ${ }^{5}$ has highlighted the need for coordinated antibiotic programmes to treat donovanosis (and other genital ulcerative diseases) and hence reduce the transmission of HIV. Azithromycin could be the ideal antibiotic for such programmes. The drug penetrates genital tract tissue ${ }^{8}$ and concentrates within macrophages, ${ }^{11}$ which are the cells infected by Cal granulomatis, ${ }^{1}$ then is slowly released. ${ }^{8}$ This pharmacokinetic profile makes effective and convenient treatment protocols 
possible. Out-patients with small lesions could receive supervised weekly doses (such as regimen A) from community health workers. Alternatively, individuals with extensive disease could be admitted briefly to hospital for dressings to the lesion(s) and daily azithromycin treatment (for example regimen B), which would produce therapeutic tissue levels lasting several weeks after discharge. Furthermore, patients with donovanosis may have multiple STDs. Azithromycin is the treatment of choice for chlamydia, is effective against gonorrhoea and chancroid, and has anti-syphilitic activity. ${ }^{612} 13$

Since completion of the pilot study, azithromycin has been recommended as the first-line therapy for donovanosis in the Top End of the Northern Territory. A further 17 patients who met an expanded case definition (one not necessarily relying on histological confirmation) have been successfully treated with azithromycin including a number who failed to respond to multiple courses of antibiotics prescribed over several years. Patient acceptance of azithromycin (and hence compliance) has been a feature of the new therapeutic protocol, with several patients spontaneously commenting on the rapid improvement in symptoms following the first dose of azithromycin and on the lack of sideeffects associated with the medication. The use of azithromycin has also countered the "therapeutic-nihilism" which is so often seen in staff involved in the treatment of donovanosis and which is another of the barriers to control of the disease.

Cost may be the only impediment to using azithromycin as it is significantly more expensive than "standard" antibiotics. Arrangements could be made between the manufacturer and national and international health agencies to make azithromycin available to donovanosis- endemic developing countries and indigenous populations. An azithromycin treatment programme in conjunction with communitybased STD education, could eliminate the significant morbidity caused by donovanosis and prevent the increased HIV transmission associated with this neglected genital ulcerative disease.

We thank Dr Leo Santos for performing the histopathological investigations, Dr Bart Currie for his helpful suggestions, and other Northern Territory health staff for assistance in enrolling and reviewing patients. The study was funded in part by Pfizer Pty Limited. IB was also supported by the Menzies School with funds from the Northern Territory government and the Howard Hughes Medical Institute.

1 Richens J. The diagnosis and treatment of donovanosis (granuloma inguinale). Genitourin Med 1991;67:441-52. 2 Mein J, Patel A, Bowden FJ. Surveillance of Donovanosis in the Northern Territory. Venereology 1995:8:16-20.

3 Wasserheit JN. Epidemiological synergy. Interrelationships between human immunodeficiency virus infection and other STDs. Sex Transm Dis 1992;19:61-77.

4 O'Farrell N, Windsor I, Becker P. HIV-1 infection among heterosexual attenders at a sexually transmitted diseases
clinic in Durban. $S$ Afr Med $\mathcal{f} 1991 ; 80: 17-20$.

5 O'Farrell N. Global eradication of donovanosis: an opportunity for limiting the spread of HIV-1 infection. tunity for limiting the spread

6 Bastian I, Bowden F, Mein J, et al. Molecular characterisation of the donovanosis organism. IUVDT World STD/AIDS Congress, 19-23 March 1995, Singapore. Abstract FP8, p. 87.

7 Merianos A, Gilles M, Chuah J. Ceftriaxone in the treatment of chronic donovanosis in central Australia. Genitourin Med 1994;70:84-89.

8 Peters D, Freidel H, McTavish D. Azithromycin: a review of its antimicrobial activity, pharmacokinetic properties and clinical efficacy. Drugs 1992;44:750-99.

9 Sehgal V, Shyamprasad A, Beohar P. The histopathological diagnosis of donovanosis. $B r \mathcal{F}$ Venereal Dis 1984;60: 45-7.

10 Goldberg J. Studies on granuloma inguinale V. Isolation of a bacterium resembling Donovania granulomatis from faeces of a patient with granuloma inguinale. $\mathrm{Br} \mathcal{F}$ Venereal Dis 1962;38:99-102.

11 Gladue $R$, Bright $G$, Isaacson $R$, Newborg $M$. In vitro and in vivo uptake of azithromycin (CP-62,993) by phagocytic cells: possible mechanism of delivery and release at sites of infection. Antimicrob Agents Chemother 1989;33: 277-82.

12 Bowden F, Farmer B, Bullen J, Chamberlain V, Bastian I. Azithromycin and syphilis. Genitourin Med 1995;71: 196-7.

13 Verdon MS, Handsfield HH, Johnson RB. Pilot study of azithromycin for treatment of primary and secondary syphilis. Clin Infect Dis 1994;19:486-8. 